\title{
BMJ Open Are population trends in high-risk alcohol consumption in smokers associated with trends in quit attempts and quit success? A time-series analysis
}

\author{
Emma Beard (D , ${ }^{1}$ Jamie Brown, ${ }^{1,2}$ Robert West, ${ }^{1}$ Susan Michie ${ }^{3}$
}

To cite: Beard E, Brown J, West R, et al. Are population trends in high-risk alcohol consumption in smokers associated with trends in quit attempts and quit success? A time-series analysis. BMJ Open 2020;10:e034262. doi:10.1136/ bmjopen-2019-034262

- Prepublication history for this paper is available online. To view these files, please visit the journal online (http://dx.doi. org/10.1136/bmjopen-2019034262).

Received 12 September 2019 Revised 18 February 2020 Accepted 06 March 2020
Check for updates

(C) Author(s) (or their employer(s)) 2020. Re-use permitted under CC BY. Published by BMJ.

${ }^{1}$ Research Department of Behavioural Science and Health, University College London, London, UK

${ }^{2}$ SPECTRUM Collaboration, London, United Kingdom

${ }^{3}$ Clinical, Educational and Health Psychology, University College London, London, UK

Correspondence to Dr Emma Beard; e.beard@ucl.ac.uk

\section{ABSTRACT}

Objectives Monthly changes in the prevalence of high-risk drinking and smoking in England appear to be positively correlated. This study aimed to assess how far monthly changes in high-risk drinking were specifically associated with attempts to stop smoking and the success of quit attempts.

Design Data were used from the Alcohol and Smoking Toolkit Studies between April 2014 and June 2018.

These involve monthly household face-to-face surveys of representative samples of $\sim 1800$ adults.

Setting England.

Participants Data were aggregated on 17560 past-year smokers over the study period.

\section{Primary and secondary outcome}

measures Autoregressive integrated moving average with exogenous input (ARIMAX) modelling was used to assess the association over time between monthly prevalence of high-risk drinking among smokers and (a) prevalence of attempts to quit smoking and (b) prevalence of successful quit attempts in those attempting to quit. Bayes factors (BF) were calculated to compare the null hypothesis with the hypothesis of an effect sufficiently large $(\beta=0.6)$ to explain the established association between overall prevalence in smoking and high-risk drinking.

Results No statistically significant associations were found between monthly changes in prevalence of high-risk drinking among smokers and attempts to quit smoking ( $\beta=0.156,95 \% \mathrm{Cl}-0.079$ to $0.391, p=0.194)$ or quit success $(\beta=0.066,95 \% \mathrm{Cl}-0.524$ to $0.655, \mathrm{p}=0.827)$. $\mathrm{BF}$ indicated that the data were insensitive but suggested there is weak evidence for the null hypothesis in the case of both quit attempts $(\mathrm{BF}=0.80)$ and quit success (BF=0.53).

Conclusions Monthly changes in prevalence of high-risk alcohol consumption in England are not clearly associated with changes in quit attempt or quit success rates.

\section{BACKGROUND}

In England, around $15 \%$ of the population are smokers and 20\% drink alcohol at highrisk levels, that is, levels which are likely to cause harm. ${ }^{12}$ Both are associated with a number of preventable conditions and appear to have an accumulative effect on the risk of mortality. ${ }^{3}$ The association between
Strengths and limitations of this study

This is the first time-series study to assess how far monthly changes in high-risk drinking are associated with attempts to stop smoking and the success of quit attempts.

- This study uses a large representative sample of the population in England.

- In countries with weaker tobacco control, different effects may be observed.

- Data are observational and so strong conclusions regarding cause and effect cannot be made.

high-risk drinking and smoking has been well established at an individual level. Highrisk drinkers are substantially more likely to smoke $^{4-8}$ and smokers who report starting a quit attempt also report lower alcohol consumption. $^{9}{ }^{10}$ Attempts to quit smoking are also less successful among those with an alcohol use disorder. ${ }^{11-13}$ Such associations may arise by a number of mechanisms. For example, smokers drinking at high-risk levels may follow advice that it is important to restrict alcohol consumption when they quit, ${ }^{9}{ }^{14-16}$ alcohol and smoking appear to provide cues to lapses for the other and there may be pharmacological interactions between nicotine and alcohol. ${ }^{17-19}$ This is contrary to the popular notion of self-medication and reward seeking with people deprived of cigarettes compensating by increasing their use of alcohol. ${ }^{20}$

It is important to identify whether similar patterns exist at a population level. An association in either direction could mean that policies that reduce smoking prevalence may have the added benefit of reducing high-risk drinking or vice versa. In England, since 2014, monthly data have been gathered on highrisk drinking, smoking status, attempts to quit smoking and quit success. ${ }^{21}$ Recently, we used these data to examine population-level 
associations over time between smoking and high-risk drinking and showed that monthly changes in prevalence of smoking in England were associated positively with prevalence of high-risk drinking. However, there were no significant associations between motivation to stop and motivation to reduce alcohol consumption or attempts to quit smoking and attempts to reduce alcohol consumption. ${ }^{22}$ We found the combination of results surprising and suggested that the association with overall prevalence may be related to an unmeasured variable that accounted for the change in both smoking and high-risk drinking. Alternatively, the failure to find an overall association between motivation and attempts for each behaviour may be an issue of power when focussing on the global association between subsamples that represented only a fifth of the overall sample.

This study attempted to resolve this apparent contradiction and explore the previously identified positive association between prevalence of smoking and prevalence of high-risk drinking. We relied on the assessment of trends between more specific outcomes expected to be more strongly related, if the identified association between the changes in the overall prevalence of smoking and highdrinking was causal. Specifically, we will assess whether changes in trends of excessive alcohol consumption among smokers are associated with trends in attempts to quit smoking and quit success. If no association is found, this would support the conclusion of a third unmeasured variable associated with both smoking and high-risk drinking.

This study addressed the following research questions:

1. Is there an association in England between increases or decreases in monthly prevalence of high-risk drinking among smokers and attempts to quit smoking?

2. Is there an association in England between increases or decreases in monthly prevalence of high-risk drinking among smokers and quit success rates?

\section{METHODS}

\section{Study design}

Data were used from the Smoking and Alcohol Toolkit Studies (STS and ATS) collected between April 2014 and June 2018. The STS and ATS are ongoing studies that involve a series of monthly cross-sectional household, face-to-face, computer-assisted surveys of representative samples of 1800 adults in England aged above 16. Thus, the same participants take part in both surveys. The respondents are recruited using a type of random location sampling, which is a hybrid between random probability and simple quota sampling. England is first split into over 170000 'output areas', comprising of approximately 300 households. These areas are then stratified according to A Classification Of Residential Neighbourhoods (ACORN) characteristics and geographic region (http://www.caci.co.uk/acorn/) and are randomly allocated to interviewers. Interviewers visit households within their allocated locality starting at a random point in the area. One member per household, chosen based on who the interviewer judge would best fulfil their quota requirements, is interviewed until interviewers achieve local quotas designed to minimise differences in the probability of participation. Participants appear to be representative of the population in England, having similar sociodemographic composition and smoking characteristics to large national surveys based on probability samples such as the Health Survey for England, ${ }^{23}$ while drinking characteristics also appear similar at a regional level to other national surveys. ${ }^{24}$ For further details, see: www. smokinginengland.info and www.alcoholinengland.info and the published protocols. ${ }^{2123}$

\section{Participants}

Data were collected on 88122 participants over the study period. Of these, $19.9 \%$ (95\% CI 19.7 to $20.2 \mathrm{n}=17560$ ) reported that they had smoked in the past year. Fortyseven per cent of past-year smokers $(n=8097)$ were men, $18.9 \%(\mathrm{n}=3272)$ were aged $16-24,19.7 \%(\mathrm{n}=3416)$ were aged $25-34,16.2 \%(\mathrm{n}=2804)$ were aged $35-44,17.0 \%$ $(\mathrm{n}=2946)$ were aged $45-54,14.6 \%(\mathrm{n}=2521)$ were aged $55-64$ and $13.7 \%(n=2371)$ were aged above 65 . Finally, $59.4 \%$ ( $\mathrm{n}=10286$ ) were in manual occupations. Data from these participants were aggregated monthly and this forms the basis of the sample in this paper.

\section{Measures}

\section{Input series}

Participants were asked whether they smoked or had smoked cigarettes (including hand rolled) daily or nondaily in the past year and to complete the Alcohol Use Disorders Identification Test (AUDIT). ${ }^{25}$ The AUDIT identifies people who could be classified as dependent, harmful or hazardous drinkers and has demonstrated validity, high internal consistency and good test-retest reliability across gender, age and cultures. ${ }^{26-29}$ Those scoring 8 or more were classed as high-risk drinkers. This is a common threshold for high-risk drinkers. ${ }^{27}$ 30-32 The prevalence of high-risk alcohol consumption among smokers in each month was obtained by counting the number smokers reporting an AUDIT score greater than or equal to 8 .

\section{Output series}

Past-year smokers were then asked:

1. 'How many serious attempts to stop smoking have you made in the last 12 months? By serious attempt I mean you decided that you would try to make sure you never smoked again. Please include any attempt that you are currently making and please include any successful attempt made within the last year'.

2. 'How long did your most recent serious quit attempt last before you went back to smoking?'

The monthly prevalence of quit attempts was calculated as the number of respondents who reported having made one or more quit attempts in the past 12 months divided by the number of past-year smokers. The success rate in 
each quarter was calculated as the number of respondents reporting that they were still not smoking divided by the number reporting having made a quit attempt.

\section{Covariates}

Past-year smokers' socioeconomic status was assessed by social grade measured using the British National Readership Survey Social Grade Classification $\mathrm{Tool}^{27}$ : $\mathrm{AB}$ (higher managerial, administrative or professional), C1 (supervisory or clerical and junior managerial, administrative or professional), C2 (skilled manual workers), D (semiskilled and unskilled manual workers) and $\mathrm{E}$ (casual or lowest grade workers, pensioners, and others who depend on the welfare state for their income). The prevalence of smokers in lower social grades in each quarter was calculated as the proportion of past-year smokers who reported being in C2, D and E. Past-year smokers were also asked their age, with a mean estimated each month.

\section{Analysis}

The analysis plan, data and syntax were preregistered on the Open Science Framework (https://osf.io/384gx/). An amendment was made to the analysis plan following reviewer comments to also adjust for sociodemographic variables. Variables can only be included in ARIMAX models at the aggregated level and must vary sufficiently over the study period. ${ }^{33}$ There was insufficient variation in gender and ethnicity over the period but there was sufficient variation in mean age and the proportion of those in lower social grades, which were included. Studies have shown an increase in the age of smokers over time t $^{34}$ and socioeconomic status is a strong predictor of quitting activity. ${ }^{35} 36$

Cases with missing data on either smoking or drinking variables were classified as missing in calculating the prevalence figures: smoking status $(\mathrm{n}=55 ; \%=0.1)$, high-risk drinking status among smokers $(\mathrm{n}=202 ; \%=1.2)$ and quit attempts among smokers $(\mathrm{n}=562 ; \%=3.2)$. All data were analysed in R studio.

Data were weighted (see Fidler et $a t^{23}$ for further details) to match the population in England and analysed using autoregressive integrated moving average with exogenous input (ARIMAX) modelling to assess the association between prevalence of high-risk drinking among smokers and (1) prevalence of attempts to quit smoking and (2) prevalence of successful attempts to quit smoking among those having made a quit attempt. ARIMAX is an extension of ARIMA analysis, which produces forecasts based on prior values in the time series (autoregressive terms; AR) and the errors made by previous predictions (moving average terms; MA). We followed a standard ARIMAX modelling approach. ${ }^{37}$

The ARIMAX assumption of weak exogeneity was met: past prevalence of quit attempts $(\mathrm{p}=0.747)$ and quit success $(p=0.999)$ did not statistically predict the future prevalence of high-risk drinking among smokers. No outliers were identified in any of the series using an approach based on that described by Chen and Liu. 3839
To stabilise the variance, the series was log transformed. The augmented Dickey-Fuller test and visual inspection of the plots indicated that first-order differencing was required for both time series. First-order differencing involves calculating the change between one observation and the next. No additional seasonal differencing was required. ${ }^{40}$

The autocorrelation and partial autocorrelation functions were examined to determine the non-seasonal MA and AR terms. These suggested an $\operatorname{ARIMAX}(0,1,1)$ model for the time series predicting both prevalence of quit attempts and prevalence of quit success. This was confirmed by comparing models with different specifications using the Akaike information criterion (AIC). To identify the most appropriate transfer function for the continuous explanatory variables, the sample crosscorrelation function was checked and models with varying distributed lags compared using the AIC. This suggested a lag of 0 when predicting the prevalence of quit attempts and predicting the prevalence of quit success, thus only current values and not lagged (past period) values of the input series were used to predict current values of the output series. In our previous study, prevalence of smoking was found to be associated with high-risk drinking with a distributed lag of $2 .{ }^{22}$ Thus, additional sensitivity analyses were run with the output series lagged by an order of 2 , that is, the time base was shifted back by 2 months.

The Ljung-Box test for white noise showed that the residuals for both fitted models were free of serial correlation. A number of additional model checks were also made. First, the autocorrelation terms included in the model were checked for their statistical significance. Second, it was determined whether the model residuals were normally distributed, random and independent. Finally, that the inclusion of the MA term conformed to the bounds of invertibility, that is, its value was $<1 .{ }^{3738}$

Bayes factors (BFs) were derived for non-significant findings using an online calculator to disentangle whether there is evidence for the null hypothesis of no effect (BF $<1 / 3 \mathrm{rd}$ ) or the data are insensitive (BF between 1/3rd and 3) ${ }^{4142}$ A half-normal distribution was assumed with a percentage change in the outcomes of interest for every percentage increase in the input series of $0.6 \%$. This is on the basis of a previous study showing that smokers who had made a quit attempt were around $40 \%$ less likely to report that they were high-risk drinkers. ${ }^{9}$ Strengthening the Reporting of Observational Studies in Epidemiology (STROBE) guidelines for the reporting of observational studies were followed throughout. ${ }^{43}$

\section{PATIENT INVOLVEMENT}

Neither patients were involved in setting the research question or the outcome measures nor were they involved in developing plans for recruitment, design or implementation of the study. No patients were asked to advise on interpretation or writing up of results. There are no plans 

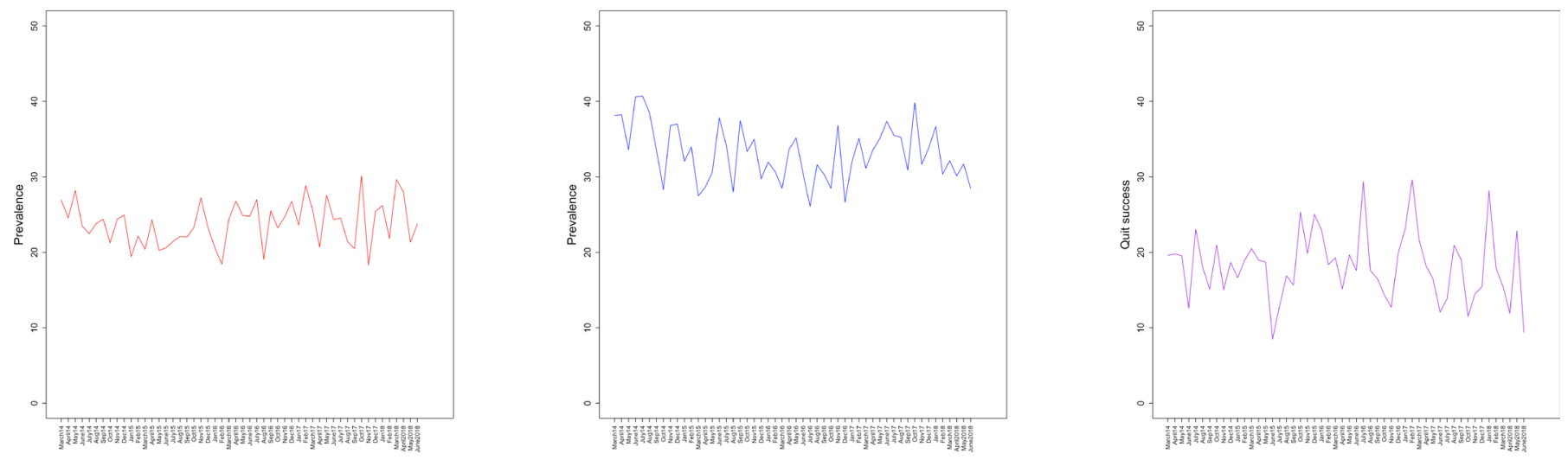

Figure 1 Prevalence of (A) high-risk drinking, (B) attempts to quit smoking and (C) quit success.

to disseminate the results of the research directly to study participants or any specific patient community.

\section{RESULTS}

Figure 1 shows the raw time-series data from 2014 to 2018. Prevalence of high-risk drinking among smokers declined from $26.9 \%$ (95\% CI 22.3 to 32.0 ) in 2014 to $23.7 \%$ (95\% CI 19.3 to 28.9) in June 2018. Attempts to quit smoking also declined from $38.1 \%$ (95\% CI 32.7 to 43.7 ) to $28.5 \%$ (95\% CI 23.6 to 33.9) and quit success from $19.6 \%$ (95\% CI 13.2 to 27.9 ) to $9.4 \%$ (95\% CI 4.5 to 18.0 ) in June 2018.

Table 1 shows the results of the ARIMAX models assessing the association between prevalence of high-risk drinking among smokers and (1) quit attempts and (2) quit success. The findings were inconclusive as to whether any associations were present. BFs suggested that there is anecdotal evidence for the null hypothesis that prevalence of high-risk drinking among smokers is not associated with prevalence of quit attempts and quit success. Findings were similar when a 2-month back shifted lag was used for prevalence of quit attempts and quit success. Adjusting for age and social grade did not change the findings (table 2).

\section{DISCUSSION}

To the best of our knowledge, this is the first empirical study to estimate the population association between high-risk drinking among smokers and attempts to quit smoking and the success of those attempts. There was weak evidence that there was no substantial association between changes in the prevalence of high-risk drinking and quit attempts and quit success.

These findings appear to be at odds with individuallevel studies, which suggest that smokers with an alcohol use disorder are less likely to attempt and succeed in stopping smoking. ${ }^{12} 13$ Alcohol consumption during attempts at smoking cessation is also associated with a greater risk of relapse. ${ }^{14}$ As a result, smokers are often advised to lower their alcohol consumption when they attempt to quit smoking. ${ }^{9}$ Of course, it remains plausible that

Table 1 Estimated percentage point changes in proportion of quit attempts and proportion of quitters who met criteria for quit success during the study period, based on the autoregressive integrated moving average with exogenous input (ARIMAX) models

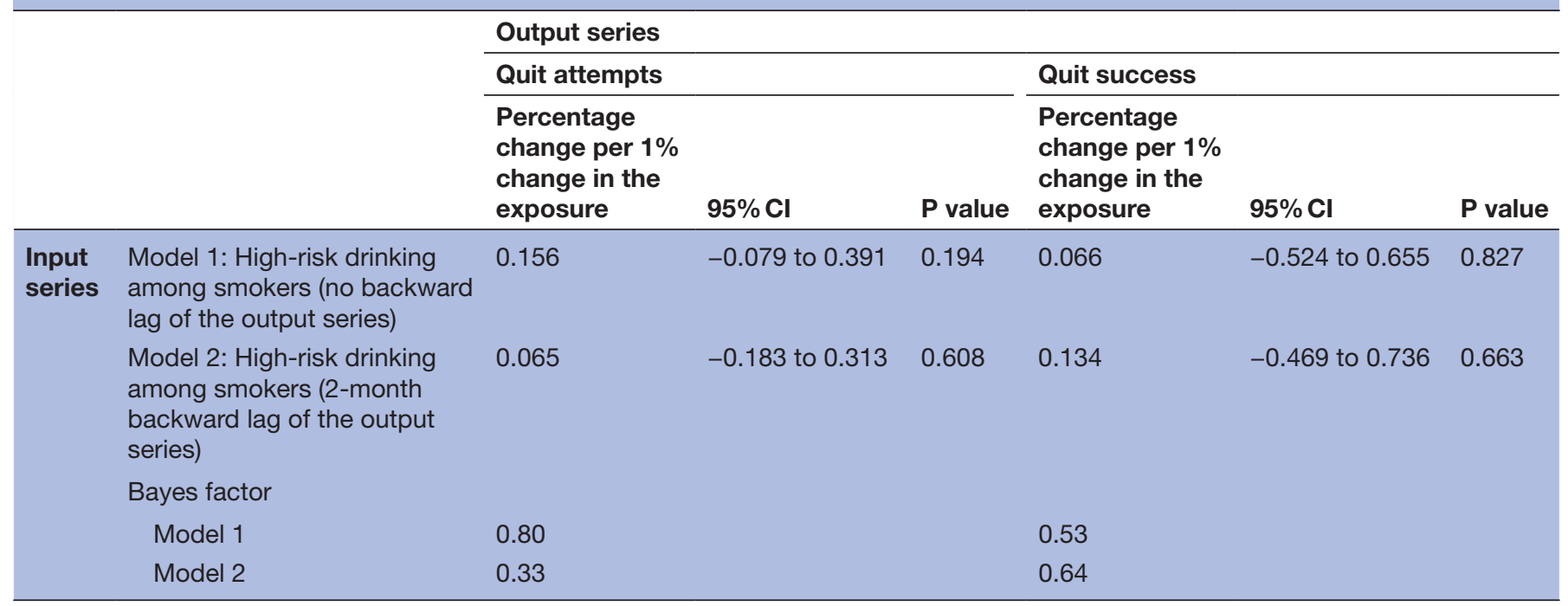


Table 2 Estimated percentage point changes in proportion of quit attempts and proportion of quitters who met criteria for quit success during the study period, based on autoregressive integrated moving average with exogenous input (ARIMAX) models - adjusted age and socialeconomic status

\begin{tabular}{|c|c|c|c|c|c|}
\hline \multicolumn{6}{|l|}{ Output series } \\
\hline Quit attempts & & & Quit success & & \\
\hline $\begin{array}{l}\text { Percentage } \\
\text { change per } 1 \% \\
\text { change in the } \\
\text { exposure }\end{array}$ & $95 \% \mathrm{Cl}$ & P value & $\begin{array}{l}\text { Percentage } \\
\text { change per } 1 \% \\
\text { change in the } \\
\text { exposure }\end{array}$ & $95 \% \mathrm{Cl}$ & $P$ value \\
\hline 0.040 & -0.214 to 0.294 & 0.758 & 0.168 & -0.489 to 0.825 & 0.616 \\
\hline 0.030 & -0.229 to 0.289 & 0.822 & 0.132 & -0.549 to 0.814 & 0.703 \\
\hline
\end{tabular}

high-risk drinking among smokers may still be associated with a small effect on mean population prevalence of quit attempts and their success, but it was not possible to detect this in the current study. An association may also be masked by factors impacting at a population level, which were not accounted for in the current study. Although we are unaware of any major population-level interventions or other events during the study period which may have affected the associations under investigation, we cannot rule out residual confounding. There may also be some statistical bias due to the loss of power and sensitivity that comes with the aggregating data. Prevalence of high-risk drinking among smokers will also be somewhat noisier than if prevalence was also assessed among non-smokers, given the smaller sample size involved in the estimation.

These findings suggest that the previously identified positive association between prevalence of smoking and prevalence of high-risk drinking is unlikely to be causal, whereby smokers attempting to quit, and those succeeding, also reduce their alcohol intake. ${ }^{22}$ Although it remains possible that use of alcohol by smokers impacts on other key indices including longer term abstinence, the small proportion of smokers who relapse long term (ie, after a year) could not account for the size of association noted. It may instead be that overall prevalence is related to an unmeasured variable, perhaps economic factors and sociocultural events, that accounts for the change in both smoking and high-risk drinking. For example, in recent years, taxation on cigarettes and alcohol has increased linearly, driving down sales of both. ${ }^{45}$ There have also been substantial fluctuations in average household income since 2013, which have been shown to independently affect smoking and alcohol consumption. ${ }^{46-48}$ Sporting events such as the Olympics may also concurrently increase alcohol and tobacco intake as they are celebratory occasions. Mass media campaigns may also play role, simultaneously promoting attempts to quit smoking and the adoption of a healthier lifestyle by reducing alcohol intake. ${ }^{49}$
A strength of this study is the use of a large representative sample of the population in England. Several limitations need to be considered. First, the ATS required participants to recall their alcohol consumption and attempts to quit smoking which is likely to have been somewhat inaccurate due to recall bias and social desirability. For example, it has been found that a large proportion of unsuccessful quit attempts fail to be reported, particularly if they only last a short time or occurred long ago. ${ }^{50}$ However, social pressure in population surveys tends to be low, and so it is generally considered acceptable to rely on self-reported data. ${ }^{51}$ Secondly, these findings may not generalise to other countries. England has a strong tobacco-control climate. In countries with weaker tobacco control or different alcohol control policies, different effects may be observed. Thirdly, this paper did not consider the impact of changes in excessive alcohol consumption prevalence on the length of quit success, being defined as having made a quit in attempt in the last 12 months and still reporting not smoking. This will be an important area for future research as more data are accumulated to provide adequate power. Finally, although there can be no individual-level confounding in population trend data, there is a possibility of population-level confounding, such as introduction of policies that may affect quitting rates. However, we were unable to identify any such population policies occurring during the study period that may have confounded the results.

\section{CONCLUSION}

These findings suggest that the previously identified positive association between prevalence of smoking and prevalence of high-risk drinking is unlikely to be causal, whereby smokers attempting to quit, and those succeeding, also reduce their alcohol intake. Instead, it may be that overall prevalence is related to an unmeasured third variable such as economic factors and sociocultural events. 
Twitter Jamie Brown @jamiebrown10

Contributors EB, JB, SM and RW wrote the first draft of the manuscript and conducted the analysis. All other authors commented on this draft and contributed to the final version. All authors read and approved the final manuscript.

Funding The Smoking Toolkit Study is currently primarily funded by Cancer Research UK (C1417/A14135; C36048/A11654; C44576/A19501) and has previously also been funded by Pfizer, GSK and the Department of Health. The ATS is currently funded by the NIHR School for Public Health Research (SPHR) (SPHRSWP-ALC-WP5). SPHR is a partnership between the Universities of Sheffield; Bristol; Cambridge; Exeter; UCL; The London School for Hygiene and Tropical Medicine; the LiLaC collaboration between the Universities of Liverpool and Lancaster and Fuse; The Centre for Translational Research in Public Health, a collaboration between Newcastle, Durham, Northumbria, Sunderland and Teesside Universities. JB's post is funded by CRUK (C1417/A14135). RW is funded by Cancer Research UK (C1417/ A14135). EB is funded by the NIHR SPHR (SPHR-SWP-ALC-WP5) and CRUK also provides support (C1417/A14135)

Disclaimer The views expressed are those of the authors and not necessarily those of the NHS, NIHR or Department of Health. No funders had any involvement in the design of the study, the analysis or interpretation of the data, the writing of the report or the decision to submit the paper for publication.

Competing interests RW undertakes consultancy and research for and receives travel funds and hospitality from manufacturers of smoking cessation medications. RW salary is funded by Cancer Research UK. SM receives support from Cancer Research UK and the National Institute for Health Research (NIHR)'s School for Public Health Research (SPHR). EB and JB have received unrestricted research funding from Pfizer. PM's research is funded by a variety of governmental funding agencies including UKRI and NIHR.

Patient and public involvement Patients and/or the public were not involved in the design, or conduct, or reporting, or dissemination plans of this research.

Patient consent for publication Not required.

Ethics approval Ethics approval for the Smoking Toolkit Survey (STS) was originally granted by the UCL Ethics Committee (ID 0498/001) and approval for the ATS was granted by the same committee as an extension of the STS (ID 2808/005). In accordance with our ethical approval, all respondents were given a written information sheet about the study and provided informed verbal consent.

Provenance and peer review Not commissioned; externally peer reviewed.

Data availability statement Data are available in a public, open access repository. The analysis plan, data and syntax were preregistered on the Open Science Framework (https://osf.io/384gx/).

Open access This is an open access article distributed in accordance with the Creative Commons Attribution 4.0 Unported (CC BY 4.0) license, which permits others to copy, redistribute, remix, transform and build upon this work for any purpose, provided the original work is properly cited, a link to the licence is given and indication of whether changes were made. See: https://creativecommons.org/ licenses/by/4.0/.

\section{ORCID iD}

Emma Beard http://orcid.org/0000-0001-8586-1261

\section{REFERENCES}

1 Office for National Statistics. Adult smoking habits in the UK: 2017 , 2018.

2 Health and Social Care Information Centre. Statistics on alcohol, England 2016, 2016. Available: https://www.gov.uk/government/ statistics/statistics-on-alcohol-england-2016

3 Kvaavik E, Batty GD, Ursin G, et al. Influence of individual and combined health behaviors on total and cause-specific mortality in men and women: the United Kingdom health and lifestyle survey. Arch Intern Med 2010;170:711-8.

4 Lasser K, Boyd JW, Woolhandler S, et al. Smoking and mental illness: a population-based prevalence study. JAMA 2000;284:260610.

5 Falk DE, Yi H-ye, Hiller-Sturmhöfel S. An epidemiologic analysis of co-occurring alcohol and tobacco use and disorders: findings from the National epidemiologic survey on alcohol and related conditions. Alcohol Res Health 2006;29:162-71.

6 Kalman D, Morissette SB, George TP. Co-morbidity of smoking in patients with psychiatric and substance use disorders. Am J Addict 2005;14:106-23.
7 Kessler RC, Chiu WT, Demler O, et al. Prevalence, severity, and comorbidity of 12-month DSM-IV disorders in the National comorbidity survey replication. Arch Gen Psychiatry 2005;62:617.

8 Compton WM, Thomas YF, Stinson FS, et al. Prevalence, correlates, disability, and comorbidity of DSM-IV drug abuse and dependence in the United States: results from the National epidemiologic survey on alcohol and related conditions. Arch Gen Psychiatry 2007;64:566-76.

9 Brown J, West R, Beard E, et al. Are recent attempts to quit smoking associated with reduced drinking in England? A cross-sectional population survey. BMC Public Health 2016;16:1-7.

10 Przulj D, Hajek P, Snuggs S, et al. Changes in alcohol consumption during a stop-smoking attempt and differences between smokers using nicotine replacement and smokers using varenicline. Nicotine Tob Res 2018;20:583-8.

11 Hughes JR, Kalman D. Do smokers with alcohol problems have more difficulty quitting? Drug Alcohol Depend 2006;82:91-102.

12 Weinberger AH, Pilver CE, Hoff RA, et al. Changes in smoking for adults with and without alcohol and drug use disorders: longitudinal evaluation in the US population. Am J Drug Alcohol Abuse 2013;39:186-93.

13 Leeman RF, McKee SA, Toll BA, et al. Risk factors for treatment failure in smokers: relationship to alcohol use and to lifetime history of an alcohol use disorder. Nicotine Tob Res 2008;10:1793-809.

14 Kahler CW, Spillane NS, Metrik J. Alcohol use and initial smoking lapses among heavy drinkers in smoking cessation treatment. Nicotine Tob Res 2010;12:781-5.

15 Gulliver SB, Kamholz BW, Helstrom AW. Smoking cessation and alcohol abstinence: what do the data tell us? Alcohol Res Health 2006;29:208-12.

16 Friend KB, Pagano ME. Smoking cessation and alcohol consumption in individuals in treatment for alcohol use disorders. J Addict Dis 2005;24:61-75.

17 Rose JE, Brauer LH, Behm FM, et al. Psychopharmacological interactions between nicotine and ethanol. Nicotine Tob Res 2004;6:133-44.

18 Field M, Mogg K, Bradley BP. Alcohol increases cognitive biases for smoking cues in smokers. Psychopharmacology 2005;180:63-72.

19 Verplaetse TL, McKee SA. An overview of alcohol and tobacco/ nicotine interactions in the human laboratory. Am J Drug Alcohol Abuse 2017;43:186-96.

20 Blume AW, Schmaling KB, Marlatt GA. Revisiting the self-medication hypothesis from a behavioral perspective. Cogn Behav Pract 2000;7:379-84.

21 Beard E, Brown J, West R, et al. Protocol for a national monthly survey of alcohol use in England with 6-month follow-up: 'the Alcohol Toolkit Study'. BMC Public Health 2015;15:230.

22 Beard E, West R, Michie S, et al. Association between smoking and alcohol-related behaviours: a time-series analysis of population trends in England. Addiction 2017;112:1832-41.

23 Fidler JA, Shahab L, West O, et al. 'The smoking toolkit study': a national study of smoking and smoking cessation in England. BMC Public Health 2011;11:479.

24 Beard E, Brown J, West R, et al. Healthier central England or NorthSouth divide? Analysis of national survey data on smoking and highrisk drinking. BMJ Open 2017;7:e014210.

25 Casswell S, Meier P, MacKintosh AM, et al. The International alcohol control (IAC) study-evaluating the impact of alcohol policies. Alcohol Clin Exp Res 2012;36:1462-7.

26 Saunders JB, Aasland OG, Amundsen A, et al. Alcohol consumption and related problems among primary health care patients: WHO collaborative project on early detection of persons with harmful alcohol consumption--I. Addiction 1993:88:349-62.

27 Saunders JB, Aasland OG, Babor TF, et al. Development of the alcohol use disorders identification test (AUDIT): WHO collaborative project on early detection of persons with harmful alcohol consumption--II. Addiction 1993;88:791-804.

28 Allen JP, Litten RZ, Fertig JB, et al. A review of research on the alcohol use disorders identification test (AUDIT). Alcohol Clin Exp Res 1997;21:613-9.

29 Hays RD, Merz JF, Nicholas R. Response burden, reliability, and validity of the CAGE, short MAST, and AUDIT alcohol screening measures. Behav Res Method Instrument Comp 1995;27:277-80.

30 Caviness CM, Hatgis C, Anderson BJ, et al. Three brief alcohol screens for detecting hazardous drinking in incarcerated women. J Stud Alcohol Drugs 2009;70:50-4.

31 National Institute on Alcohol Abuse and Alcoholism. Helping patients who drink too much: a clinician's guide. Bethesda, MD: National Institute on Alcohol Abuse and Alcoholism, 2007.

32 Kokotailo PK, Egan J, Gangnon R, et al. Validity of the alcohol use disorders identification test in college students. Alcohol Clin Exp Res 2004;28:914-20. 
33 Beard E, Marsden J, Brown J, et al. Understanding and using time series analyses in addiction research. Addiction 2019;114:1866-84.

34 Garnett C, Tombor I, Beard E, et al. Have smoking characteristics and quitting behaviour changed between 2008 and 2017 in England? A population study. Submitted.

35 Hiscock R, Judge K, Bauld L. Social inequalities in quitting smoking: what factors mediate the relationship between socioeconomic position and smoking cessation? J Public Health 2011;33:39-47.

36 Parks MJ, Kingsbury JH, Boyle RG, et al. Behavioral change in response to a statewide tobacco Tax increase and differences across socioeconomic status. Addict Behav 2017;73:209-15.

37 Box GE, Jenkins GM, Reinsel GC. Time series analysis: forecasting and control. John Wiley \& Sons, 2011.

38 Chen C, Liu L-M. Joint estimation of model parameters and outlier effects in time series. J Am Stat Assoc 1993;88:284-97.

39 López-de-Lacalle J. tsoutliers $\mathrm{R}$ package for automatic detection of outliers in time series.

40 Dickey DA, Fuller WA. Distribution of the estimators for autoregressive time series with a unit root. J Am Stat Assoc 1979;74:427-31.

41 Dienes Z. Using Bayes to get the most out of non-significant results. Front Psychol 2014;5:781.

42 Beard E, Dienes Z, Muirhead C, et al. Using Bayes factors for testing hypotheses about intervention effectiveness in addictions research. Addiction 2016;111:2230-47.

43 von Elm E, Altman DG, Egger M, et al. The strengthening the reporting of observational studies in epidemiology (STROBE) statement: guidelines for reporting observational studies. PLoS Med 2007; 4:e296.

44 Institute of Alcohol Studies. How have UK alcohol taxes changed over time? 2017. Available: http://www.ias.org.uk/Alcoholknowledge-centre/Price/Factsheets/How-have-UK-alcohol-taxeschanged-over-time.aspx

45 ASH. Timeline of changes in tobacco taxation in the UK from 1993 to the present, 2017. Available: http://ash.org.uk/information-andresources/taxation-illicit-trade/taxation/ash-analysis-of-tobacco-taxincreases-in-the-united-kingdom/

46 Cerdá M, Johnson-Lawrence VD, Galea S. Lifetime income patterns and alcohol consumption: investigating the association between long- and short-term income trajectories and drinking. Soc Sci Med 2011;73:1178-85.

47 Perelman J, Alves J, Pfoertner T-K, et al. The association between personal income and smoking among adolescents: a study in six European cities. Addiction 2017;112:2248-56.

48 ONS. Nowcasting household income in the UK: financial year ending 2017, 2018

49 Wakefield MA, Loken B, Hornik RC. Use of mass media campaigns to change health behaviour. Lancet 2010;376:1261-71.

50 Berg CJ, An LC, Kirch M, et al. Failure to report attempts to quit smoking. Addict Behav 2010;35:900-4.

51 Wong SL, Shields M, Leatherdale S, et al. Assessment of validity of self-reported smoking status. Health Rep 2012;23:D1. 